\title{
Temporomandibular Joint Disorder Associated With Migraine - Clinical Study
}

\author{
Kaur $B^{1}$, Goyal $G^{1}$, Padda $S^{1}$, Garg $S^{1}$, Kalucha $A^{1}$, Kaur $S^{1}, \operatorname{Singh} B^{2}$, Kaur $K^{3}$ \\ ${ }^{1}$ Department of Oral Medicine and Radiology, Genesis Institute of Dental Sciences and Research, Ferozepur, Punjab, India \\ ${ }^{2}$ Department of Oral Medicine and Radiology, Shri Guru Ram Das Dental College, Amritsar, Punjab, India \\ ${ }^{3}$ Consultant Oral Surgeon, Bibi Kahlon Hospital, Amritsar, Punjab, India
}

Email address:

drbhawana@yahoo.co.in (Kaur B)

\section{To cite this article:}

Kaur B, Goyal G, Padda S, Garg S, Kalucha A, Kaur S, Singh B, Kaur K. Temporomandibular Joint Disorder Associated With Migraine Clinical Study. American Journal of Health Research. Special Issue: Rethinking Temporomandibular Joint in Health and Disease: At Diagnosis and Interventional Level. Vol. 3, No. 3-2, 2015, pp. 6-9. doi: 10.11648/j.ajhr.s.2015030302.12

\begin{abstract}
Objectives: The aim of this study was to assess the prevalence of Temporomandibular Disorders (TMD), using the Research Diagnostic Criteria for TMD (RDC/TMD) in women with episodic and chronic migraine (M and CM), as well as in asymptomatic women. Method: Sample consisted of 100 women, being 40 with M and 27 with CM, identified from a headache outpatient center; we also investigated 33 women without headaches for at least 3 months (women without headache group - WHG). Assessment of TMD was conducted by a physical therapist who was blind to the headache status. Results: Our sample consisted of 100 women. The mean age was 37.18 years for WHG, 38.16 years for M and 37.83 for CM. TMD was diagnosed in 10 women from the WHG, 33 women with M group and 21 women with CM group. TMD was significantly more prevalent in women with M and CM groups, as compared to WHG (Table 1). As compared with women without headache, women with $\mathrm{M}$ and CM were significantly more diagnosed with TMD and also presented higher risk for TMD (Table 1). Diagnoses of group I were the most prevalent in all groups. Mixed diagnoses (I+II and I+II+III) were observed only in individuals with migraine, and were more common in CM than in M group. Diagnoses of groups I+III were observed in the three groups, but were more prevalent in the M group than in the other groups. TMDs from groups II and III were not observed. Conclusion: Women with migraine are more likely to have muscular and articular TMD, suggesting that both disorders might be clinically associated, which demonstrate the importance of physical therapy assessment in the multidisciplinary team.
\end{abstract}

Keywords: Temporomandibular Disorders, Orofacial Pain, Physical Therapy, Rehabilitation, RDC/TMD

\section{Introduction}

Migraine and temporomandibular disorders (TMD) are chronic diseases with shared characteristics, such as the presence, duration and severity of pain, the temporal pattern and the presence of psychiatric comorbidities. ${ }^{6,7}$ Furthermore, the severity of TMD often correlates with the severity of migraine headaches. ${ }^{8}$

Migraine is an extraordinarily common disorder. It is more prevalent in women, about $18 \%$ in women and $6 \%$ in man. It is most common during the peak productive years, between the ages of 25 and 55 years-old. Migraine experience high levels of pain and substantial disability; less than $10 \%$ of migraine report that they are able to work or function normally during their headaches ${ }^{1,2}$.

The association of TMD and frequency of headaches has been well described, suggesting that both conditions are influenced by similar predisposing factors, or that they share common peripheral and/or central mechanisms. ${ }^{4,9-11}$ Most studies, however, inferred TMD based only upon their symptoms4, and few studies used objective tools for the diagnosis of this condition. ${ }^{12}$

The Research Diagnostic Criteria for Temporomandibular Disorders (RDC/TMD) requires a standardized exam, and allows the diagnosis of TMD with three sub-diagnoses: Group I describe the muscular forms of TMD; Group II refers to TMD disk displacement; Group III encompasses TMD with arthralgia, osteoarthritis, and arthroses. ${ }^{14}$ The RDC is a well validated tool for the diagnosis and assessment of TMD, with high specificity for most subtypes. ${ }^{15-17}$

Understanding the association between TMD and migraine subtypes is important for several reasons. First, TMDs, joint 
origin, cause headache attributed to TMDs, and this is recognized as a secondary headache by the Second Edition of the International Classification of Headache Disorders (ICHD-II). ${ }^{18}$ Second, TMDs may also exacerbate preexisting primary headaches (e.g. migraine) and individuals with both conditions may suffer an additional burden when compared to those with only one. $+19,20$ Finally, determine whether the frequency of migraine influences the diagnosis of TMD. Despite physical therapy in headache is little known of the effects musculoskeletal physical therapy practice has been increasingly exploited. $^{21,22}$

Therefore, the aim of this study was to assess the prevalence of TMD, using the RDC/TMD, axis 1, in women with episodic and chronic migraine ( $\mathrm{M}$ and $\mathrm{CM})$.

\section{Method}

We assessed 40 women with M, 27 with CM and 33 women without any type of headache over the past three month (WHG), with age ranging from 20 to 55 years. Patients who had used non-steroidal anti-inflammatory drugs during the 24 hours proceeding the day of the assessment, with systemic disease or history of facial trauma were excluded from the study. We have excluded patients in a migraine attack or if they report cutaneous allodynia. These exclusions were recommended by RDC/TMD validation project. $^{23}$

The presence of TMD was assessed using the research diagnostic criteria for temporomandibular Disorders $(\mathrm{RDC} / \mathrm{TMD})^{10}$. The RDC/TMD axis I, is a clinical, noninvasive assessment that has been extensively validated and used in research ${ }^{16,17}$. The RDC/TMD is a reliable and valid diagnostic research tool developed by a consensus of experts for the development of operational diagnoses for patients with TMD. The clinical examination takes about 30 minutes, which includes the measurement of the mandible range of motion, assessment of joint and muscle pain and palpation for clicks or crepitus on movements of the mandible. Furthermore, 24 sites were assessed for manual establishment of pain thresholds: posterior, middle and anterior temporalis muscle, superior, middle and inferior masseter muscle, posterior mandibular region, submandibular region, lateral/posterior pole of the temporomandibular joint, inside the mouth, lateral pterygoid area and tendon of the temporal muscle were palpated, as described in the RDC/TMD.

\section{Results}

Our sample consisted of 100 women. The mean age was 37.18 years for WHG, 38.16 years for $\mathrm{M}$ and 37.83 for CM. TMD was diagnosed in 10 women from the WHG, 33 women with $\mathrm{M}$ group and 21 women with $\mathrm{CM}$ group. TMD was significantly more prevalent in women with $\mathrm{M}$ and $\mathrm{CM}$ groups, as compared to WHG.

As compared with women without headache, women with $\mathrm{M}$ and $\mathrm{CM}$ were significantly more diagnosed with TMD and also presented higher risk for TMD.
Diagnoses of group I were the most prevalent in all groups Mixed diagnoses (I+II and I+II+III) were observed only in individuals with migraine, and were more common in $\mathrm{CM}$ than in $M$ group. Diagnoses of groups I+III were observed in the three groups, but were more prevalent in the $\mathrm{M}$ group than in the other groups. TMDs from groups II and III were not observed.

Women with $\mathrm{M}$ and $\mathrm{CM}$ had a significantly higher number of TMD diagnoses, as compared to WHG. No between-group differences were observed in those with $\mathrm{M} v s$. CM. The number of sites that presented muscle pain on palpation was significantly higher in individuals with $\mathrm{M}$ and $\mathrm{CM}$, as compared to WHG, but no between-group difference was observed between the $\mathrm{M}$ and $\mathrm{CM}$ groups. No differences were observed regarding the number of sites that had joint pain on palpation.

\section{Discussion}

Our sample consisted of patients from a tertiary outpatient clinic, which could explain the higher frequency of TMD diagnoses in all groups, although more frequently in individuals with $\mathrm{M}$ and $\mathrm{CM}$. In our study women without headache have a prevalence of $33.3 \%$ of TMD, in agreement with other clinical studies that have reported higher prevalence rates (45 to $50 \%$ ) TMD diagnoses the signs and symptoms of TMD.

Women with migraine have not only a higher prevalence of TMD, but also a higher risk factor for developing the disease. Other studies that used the RDC/TMD also reported the association of TMD and headaches, as similar findings were observed for signs and symptoms of TMD andheadaches. ${ }^{4,12,13}$ The prevalence of TMD was not different between $\mathrm{M}$ and $\mathrm{CM}$, suggesting that TMD reflects the disease itself, not disease severity in terms of frequency and the central sensitization mechanisms of neurons in migraine patients, independently of being chronic or not, could explain such findings.

Similar to other study, most individuals with TMD (from the three groups) presented myofascial pain, although the prevalence was higher in $\mathrm{M}$ and CM vs. WHG $(\mathrm{p}<0.05) .+13$ Women with $\mathrm{M}$ and $\mathrm{CM}$ had significantly higher number of diagnoses from the muscular group (group I) as compared to disk displacements (group II) and diagnoses of arthralgia, osteoarthritis and osteoarthrosis (group III) suggesting that muscular and mixed TMD could be strictly associated with headaches.

We have not observed individuals with diagnoses from groups II and III isolated, and although mixeddiagnoses (I+II, $\mathrm{I}+\mathrm{III}$ and $\mathrm{I}+\mathrm{II}+\mathrm{III})$ were verified in the three groups, it was more common in $\mathrm{M}$ and $\mathrm{CM}$ groups. In a recent study, Gonçalvesetal.suggested that the magnitude of TMD was associated with migraine severity and individuals reporting at least one TMD symptom were more likely to experience headache syndromes than individuals without TDM. ${ }^{26}$

Three hypotheses may be suggested to explain the relationship between migraine and TMD. First, mechanisms 
of peripheral and central sensitization, involved in pain perception and pain transmission ${ }^{7}$ and activated in migraine, may spread the pain from the head to the craniocervicalregion $^{27}$, decreasing the threshold for pain in the face; accordingly, individuals would have migraine with pain in the temporomandibular region (masticatory muscles and temporomandibular joint) but not true TMD. Second, mechanisms of central sensitization would be of importance for the path ophysiology and onset of both, migraine and TMD. Third, TMD or migraine may aggravate and perpetuate the other, and individuals with migraine may have their headaches worsened by the coexistence of TMD, being the opposite true as well. ${ }^{26}$

Despite the use of the gold standard for the TMD diagnosis (RDC/TMD), we have to be cautious to interpret our results. First, the RCD/TMD seems to over-diagnose problems of group I, and a possible explanation could be to the disproportion between the number of examined sites on muscles (20 sites) and joints (4 sites). It may be why the muscular groups are overrepresented in relation to the joint groups, ${ }^{30}$ yielding to the over-diagnosis of myofascialpain31. One alternative to correct this limitation of the RDC/ TMD would be to exclude muscle sites that are difficult to access (lateral pterygoid, temporal tendon, posterior region of the mandible, and submandibular region) ${ }^{32}$. Sensitivity analyses were performed by removing these tree muscle points from data and there were no differences in diagnostic rates.

The increased sensitivity in the pericranial muscles (including the masticatory muscles) of migraineurs suggest the importance and needs of multidisciplinary assessments, including the physical therapist. Furthermore, headache patients may also benefit from TMD examination, especially those who do not respond to the usual headache management.

\section{Conclusion}

TMD and migraine are clinically associated. Women with migraine are more likely to have TMD when compared to women without headache, both for the muscular and joint diagnoses. They are also more likely to have increased number of tender points, highlighting the importance of the physical therapist in the assessment of these patients.

\section{References}

[1] Lipton RB, Stewart WF, Diamond S, Diamond ML, Reed M. Prevalence and burden of migraine in the United States: data from the American Migraine Study II. Headache. 2001;41(7):646-57.

[2] Queiroz LP, Peres MFP, PiovesanEJ, Kowacs F, Ciciarelli MC, Souza JA, et al. A nationwide population-based study of migraine in Brazil. Cephalalgia. 2009;29(6):642-9.

[3] LeResche L. Epidemiology of temporomandibular disorders: implications for the investigation of etiologic factors. Crit Rev Oral Biol Med. 1997;8(3):291-305.

[4] Ciancaglini R, Radaelli G. The relationship betwwenn headache and symptoms of temporomandibular disorder in the general population. J Dent. 2001; 29(2):93-8.

[5] Lipton RB, Bigal ME. Headache as a real disease. Headache. 2008;48(5):707-10.

[6] Merrill RL. Or ofacial pain mechanisms and their clinical application. Dent Clin North Am. 1997;41(2):167-88.

[7] Sarlani E, Greenspan JD. Why look in brain for answers to temporomandibular disorder pain? Cells Tissues Organs. 2005;180(1):69-75.

[8] LiljeströmMR, Jämsä A, Le Bell Y, Alanen P, Anttila P, Metsähonkala L, et al. Signs and symptoms of temporomandibular disorders in children with different types of headache. Acta Odontol Scand. 2001;59(6):413-7.

[9] Rantala M A, Ahlberg J, Suvinen TI, Nissinen M, Lindholm $\mathrm{H}$, Savolainen A, et al. Temporomandibular joint related painless symptoms, orofacial pain, neck pain headache, and psychosocial factors among non-patients. ActaOdonltol Scand. 2003;61(4):217-22.

[10] Storm C, Wänman A. Temporomandibular disorders, headache, and cervical pain among females in a Sami population. ActaOdontol Scand. 2006;64(5):319-25.

[11] Moreno BGD, Maluf SA, Marques AP, Crivello-Junior O. Clinical and quality-of-life assessment among women with temporomandibular disorder. Rev Bras Fisioter. 2009;13(3):210-4. http://dx.doi.org/10.1590/

S1413.355.5200900.500.0028

[12] Glaros AG, Urban D, Locke J. Headache and temporomandibular disorders: evidence for diagnostic and behavioural overlap. Cephalalgia. 2007;27(6):542-9.

[13] Ballegaard V, Thede-Schmidt-Hansen P, Svensson P, Jensen R. Are headache and temporomandibular disorders related? A blinded study. Cephalalgia. 2008;28(8):832-41. http://dx.doi.org/10.1111/j.1468-2982.2008.01597

[14] Dworkin SF, LeResche L. Research diagnostic criteria for temporomandibular disorders: review, criteria, examinatios and specificatios, critique. J CraniomandibDisord. 1992;6(4):301-5.

[15] Chaves TC, Oliveira AS, Bevilaqua-Grossi D. Principaisinstrumentosparaavaliação da disfunçãotemporomandibular, parte II: critériosdiagnósticos; umacontribuiçãopara a praticaclínica e de pesquisa. Fisioter Pesqui. 2008;15(1):101-6.

[16] Dworkin SF, Sherman J, Mancl L, Ohrbach R, LeResche L, Truelove E. Reliability, validity, and clinical utility of the research diagnostic criteria for temporomandibular disorders Axis II Scales: depression, non-specific physical symptoms, and graded chronic pain. J Orofac Pain. 2002;16(3):207-20.

[17] Schmitter M, Gabbert O, Ohlmann B, Hassel A, Wolff D, Rammelsberg $\mathrm{P}$, et al. Assessment of the reliability and validity of panoramic imaging for assessment of mandibular condyle morphology using both MRI and clinical examination as the gold standard. Oral Surg Oral Med Oral Pathol Oral RadiolEndod. 2006;102(2):220-4.

[18] Subcomitê de classificação das cefaléias da SociedadeInternacional de Cefaléias. Classificação international das cefaléias. 2. ed. Trad. SociedadeBrasileira de Cefaléias. São: SegmentoFarmaEditores; 2004. 
[19] Bevilaqua-Grossi D, Lipton RB, Bigal ME. Temporomandibular disorders and migraine chronification. Curr Pain Headache Rep. 2009;13(4):314-8.

[20] Bevilaqua-Grossi D, Chaves TC, Gonçalves MC, Moreira VC, Canonica AC, Florencio LL, et al. Pressure pain threshold in the craniocervical muscles of women with episodic and chronic migraine: a controlled study. ArqNeuropsiquiatr. 2011;69(4):607-12.

[21] Fernández-de-las-Peñas C, Cleland JA, Palomeque-del-Cerro L, Caminero AB, Guillem-Mesado A, Jiménez-García R. Development of a clinical prediction rule for identifying women with tension-type headache who are likely to achieve short-term success with joint mobilization and muscle trigger point therapy. Headache. 2011;51(2):246-61. http://dx.doi. org/10.1111/j.1526-4610.2010.01789.x

[22] MorelliJGS, Rebelatto JR. The effectiveness of manual therapy in individuals with headaches, with and without cervical degeneration: analysis of six cases. Rev Bras Fisioter.
2007; 11(4):325-9. http://dx.doi.org/10.1590/

S1413.355.5200700.040.0013

[23] Schiffman E, Ohrbach R, Truelove E. RDC/TMD validation project. 2010. Cited: 26/11/03. Available from: http://www.rdc-tmdinternational.org/OtherResources/ ResearchProtocols.aspx

[24] Casanova-Rosado JF, Medina-Solís CE, Vallejos- Sánchez AA, Casanova-Rosado AJ, Hernández-Prado B, Ávila-Burgos L. Prevalence and associated factors for temporomandibular disorders in a group of Mexican adolescents and youth adults. Clin Oral Investig. 2006;10(1):42-9.

[25] BonjardimLR, Lopes-Filho RJ, Amado G, Albuquerque RLC, GonçalvesSRJ. Association between symptoms of temporomandibular disorders and gender, morphological occlusion, and psychological factors in a group of university students. Indian J Dent Res. 2009;20(2):190-4.

[26] Gonçalves DAG, Speciali JG, Jales LCF, Camparis CM, Bigal ME. Temporomandibular symptoms. 